\title{
Semipermeable Dressings Improve Epidermal Barrier Function in Premature Infants
}

\author{
ANTHONY J. MANCINI, SHARON SOOKDEO-DROST, KATHI C. MADISON, \\ BRUCE R. SMOLLER, AND ALFRED T. LANE
}

Departments of Dermatology [A.J.M., S.S.-D., B.R.S., A.T.L.], Pathology [B.R.S.], and Pediatrics [A.T.L.|, Stanford University School of Medicine, Stanford, California 94305-5486, and Department of Dermatology [K.C.M.], University of Iowa Hospitals and Clinics, Iowa City, Iowa 52242-1090

ABSTRACT

\begin{abstract}
Infants of less than $32 \mathrm{wk}$ gestation have a defective epidermal barrier, with increased skin permeability and transepidermal water loss (TEWL). We studied the effect of a nonadhesive semipermeable dressing on the epidermal barrier of premature infants and on fetal skin transplanted to nude mice. Fifteen infants with a mean estimated gestational age of $27.7 \mathrm{wk}$ and 16 human fetal skin grafts (estimated gestational age, 23-26 wk) transplanted to eight nude mice were studied. One lower leg (or skin graft) was treated and the other left untreated as a control. In the infants, TEWL was measured on control skin and treated skin (both through the dressing and after temporary dressing removal) on $\mathrm{d} 0,1,2,4$, and 7 . Bacterial and fungal cultures were also performed. In the mice, TEWL and skin blood flow were measured on $d 0,2$, and 4 . Biopsies were obtained on $\mathrm{d} 4$ for a cell proliferation assay, histology, and electron microscopy. Treated infant skin showed a consistently lower bacterial number and a significantly decreased TEWL (measured through the dressing). There was also a significantly lower TEWL on the treated side, measured after temporary dressing removal, on d 1,2, 4, and 7 ,
\end{abstract}

documenting improved epidermal barrier function. The animal study revealed decreased TEWL and a nearly 2-fold greater d-4 keratinocyte proliferation $(p=0.01)$ in treated skin and decreased blood flow on $\mathrm{d} 4$ in control skin $(p=$ $0.01)$. There was no significant difference in the volume density of membrane coating granules or the morphology of intercorneocyte spaces. It is concluded that semipermeable dressings improve epidermal barrier function without increasing bacterial or fungal colonization in premature infants, and that increased cellular proliferation is associated with improved barrier function in semipermeable dressing-treated fetal skin. (Pediatr Res 36: 306-314, 1994)
Abbreviations
TEWL, transepidermal water loss
SPD, semipermeable dressing
EGA, estimated gestational age
HFS, human fetal skin
AUC, area under the curve
BrdU, 5-bromo-2'-deoxyuridine

Premature infants of less than $32 \mathrm{wk}$ gestation have major problems associated with immaturity of their skin (1). These infants have a large surface area for body mass (2) and a compromised stratum corneum (3), reflected in high TEWL (4). TEWL is a measure of the effectiveness of the epidermal barrier and has been used to quantitate epidermal maturity (4). This measurement indicates water loss that passes through the epidermis not related to sweating.

Received November 22, 1993; accepted March 18, 1994.

Correspondence and reprint requests: Alfred T. Lane, M.D., Department of Dermatology, Stanford University School of Medicine, MSLS Bldy P204, Route 5, Stanford, CA 94305-5486.

Supported in part by National Institutes of Health General Clinical Research Center, Grant RR00081, and the Stanford Dean's Postdoctoral Fellowship Research Grant Program.
The TEWL of the premature infant decreases as the child ages. Harpin and Rutter (3) examined barrier properties in infants of 25 to 41 wk gestation. They evaluated TEWL and blanching response to phenylephrine. Their results demonstrated that earlier gestational age infants had greater skin permeability to phenylephrine and greater TEWL. In the premature infants less than $32 \mathrm{wk}$ EGA who survived the initial course, there was a rapid epidermal barrier maturation such that by $2 \mathrm{wk}$ of postnatal age they had skin permeability and TEWL nearly equivalent to values found in mature infants (3).

Occlusive dressings accelerate wound healing, a property recognized since 1962, when Winter (5) demonstrated an accelerated rate of reepithelialization in superficial wounds in swine that were treated with a polyethylene film. Many different synthetic occlusive 
dressings are now available to physicians, offering varied physicochemical properties and different degrees of permeability. These dressings have traditionally been used in the treatment of wounds such as lower leg ulcers. Although the skin of premature neonates does not show the same depth of dermal injury, the epidermal barrier is quite compromised and hence might benefit from treatment with these dressings. Previous studies using wound dressings in premature neonates have demonstrated an immediate decrease in TEWL (6-9), with no increase in bacterial or fungal colonization at sites treated with dressings over time $(8,9)$. Occlusive dressings used on standardized skin wounds in adults revealed augmented reepithelialization without a concomitant improvement in the rate of barrier recovery (10). Several of the past premature infant studies $(6,7,9)$ used adhesive dressings, which are damaging upon removal from the patient. Vernon et al. (8) used a modified dressing consisting of a small piece of nonadhesive dressing affixed centrally to a larger sheet of adhesive dressing. An advantage of using nonadhesive dressings is that barrier function can be monitored longitudinally after removal and replacement of the dressing. This is not possible with adhesive dressings, because removal itself may disrupt stratum corneum integrity and result in increased TEWL. We studied the use of an entirely nonadhesive dressing on the lower limb of 15 premature infants. We evaluated barrier function and microbial flora on treated and untreated sides over a 2-wk period.

A model has been described for transplant of fetal skin tissue to athymic nude mice (11). Grafts transplanted subcutaneously beneath the panniculus carnosus were shown to follow the same developmental time course of HFS development in utero, as long as the grafts remained in this "buried" position. When exposed to the surface, the grafts showed accelerated development (advanced over age-matched controls or control grafts that remained subcutaneous), similar to the acceleration of epidermal maturation seen in preterm infants $(11,12)$. This model provides an ideal in vivo system for the study and experimental manipulation of human epidermal differentiation and maturation, permitting more interventions than are feasible when studying premature neonates.

We used this model to study the effect of SPD on premature infant skin. Measurements of TEWL and skin blood flow (with laser Doppler blood flow analysis) were obtained. Tissue samples were then collected for histologic analysis, electron microscopy, and a BrdU cell proliferation assay.

\section{METHODS}

\section{Infant Study}

Subjects. Parents of premature infants less than $30 \mathrm{wk}$ gestational age were invited to enroll their child into the study. Gestational age was evaluated by Dubowitz criteria (13). Infants were entered into the study after informed consent was obtained from one or both parents. Fifteen infants (eight boys and seven girls) were entered; three were delivered vaginally and 12 via cesarean section. Mean gestational age was $27.7 \mathrm{wk}$ (range 24-29 wk), and mean birth weight was $1018.6 \mathrm{~g}$ (range $670-1391 \mathrm{~g}$ ). All infants were initially placed under a servo-controlled radiant warmer. Impermeable plastic "tents" or "bubble blankets," which are commonly used in this patient population in our nursery, were placed intermittently over some infants while they were under the warmer. These devices are not in uniform contact with the patient's skin, but instead are positioned above the entire body, in an effort to increase local ambient humidity and therefore decrease insensible water loss from the infant. Twelve of the 15 infants were transferred to an isolette at some point during the study. All 15 infants received parenteral antibiotics immediately after birth, and seven either continued to receive antibiotics for the duration of the study or were restarted on antibiotics at some point within the study period. All 15 infants initially received dextrose water with or without added electrolytes, and all were subsequently started on parenteral hyperalimentation at some point within the study period.

Each child in this study served as his or her own control, with one leg treated with an SPD and one leg left untreated. It was therefore unnecessary to standardize environmental conditions such as ambient humidity or room temperature, which varied from one measurement to the next.

Study design. Sheets of the polyurethane dressing Bioclusive (Johnson \& Johnson, New Brunswick, NJ), which had not yet had the adhesive applied, were provided by the manufacturer. These dressings were cut to size and sterilized with ethylene oxide before use. Within $24 \mathrm{~h}$ after birth (average time: $12.5 \mathrm{~h}$ of postnatal age), this dressing was applied to one of the lower legs of the infant. The dressing was secured just above the knee with a thin strip of adhesive Bioclusive (allowing only 1-2 mm of adhesive to be in contact with the infant's skin). The ankle was secured with a $1-\mathrm{cm}$ wide strip of Coban self-adherent wrap (3M, St. Paul, MN) (Fig. 1). The other leg remained untreated as a control. The clinical appearance of the areas of interest of both legs was judged to be equal before study entry. The SPD was placed on the leg that would least interfere with i.v. access or pulse oximetry electrode placement.

Before placement of the SPD, TEWL readings were obtained from both legs by using the Evaporimeter EP1 (ServoMed Inc., Kinna, Sweden). Immediately after placement of the dressing, TEWL was again recorded at the treated site, this time from the surface of the dressing. This was considered $\mathrm{d} 0$. All TEWL readings were measured over the gastrocnemius-soleus muscle complex on the posterior, mid-upper leg (Fig. 1). Quantitative bacterial and fungal cultures were also obtained on this day 
from both treated and control legs from the anteromedial flat surface overlying the tibia.

To evaluate the barrier maturation of the treated leg, we temporarily removed the dressing and measured the TEWL of the skin previously covered by the SPD. We were uncertain about the correct time to take this measurement after SPD removal because of the concern that the dressing would trap humidity against the skin and give spuriously high TEWL results. On 16 different occasions in the first four patients, we evaluated TEWL at various time points $(2,5,10,15,20$, and $30 \mathrm{~min})$ after dressing removal. At the time of these measurements, the dressing had been in place for at least $12 \mathrm{~h}$ and no more than $4 \mathrm{~d}$. This information was used to choose the time at which to take TEWL measurements of treated skin after temporary dressing removal on each study day.

Measurements of TEWL were obtained from the control and treated legs on $\mathrm{d} 1,2,4$, and 7 after placement of the dressing. On the treated side, TEWL was measured both from the surface of the dressing and from the underlying skin 10 min after dressing removal.

Microbiologic cultures. Quantitative cultures were performed as follows: a sterile cotton swab, moistened in $0.1 \%$ Tween-80 (a detergent) in PBS, was swabbed five times in one direction and then five times in another direction on a $1.4-\mathrm{cm}^{2}$ area of skin. The swab was then returned to the PBS and twirled several times, and the culture process repeated. Dilutions of $1 / 10,1 / 1000$, and $1 / 100000$ were prepared from the swabs, and they were then immediately spread uniformly on blood agar plates,
Sabaraud-dextrose-agar plates containing gentamicin (20 $\mathrm{mg} / \mathrm{L})$ and vancomycin $(3 \mathrm{mg} / \mathrm{L})$, and Sabaraud-dextroseagar plates containing gentamicin and vancomycin and coated with olive oil. Plates were incubated at $37^{\circ} \mathrm{C}$ and examined daily. Blood plates were incubated for $48 \mathrm{~h}$ and fungal plates for $2 \mathrm{wk}$. Gram-positive cocci were confirmed to be staphylococci by the catalase test with $\mathrm{H}_{2} \mathrm{O}_{2}$, and species was then determined to be either coagulasepositive (Staphylococcus aureus) or coagulase-negative using rabbit plasma. Budding yeasts were identified as Candida albicans by assessing for germ tube formation in rabbit plasma. Malassezia furfur was identified based on typical colonial and microscopic morphologies as well as preferential growth on the medium containing long-chain fatty acids.

Cultures were obtained in this manner on $\mathrm{d} 0,1,2,4$, and 7 . In the last six patients, the frequency of cultures was shortened to $\mathrm{d} 0,4$, and 7 .

\section{Animal Study}

Study design. HFS grafts were obtained from aborted fetuses, with EGA ranging from 18 to $22 \mathrm{wk}$. Human fetal tissue collection conformed to current recommendations of the Stanford University Human Subjects Committee. A signed informed consent was obtained. The EGA was determined from heel-to-toe fetal foot length standards (14). The skin was identified, dissected from the underlying soft tissue, and placed into RPMI medium containing $50 \mathrm{mg} / \mathrm{L}$ amphotericin $\mathrm{B}, 5 \times 10^{5} \mathrm{U} / \mathrm{L}$ penicillin, and

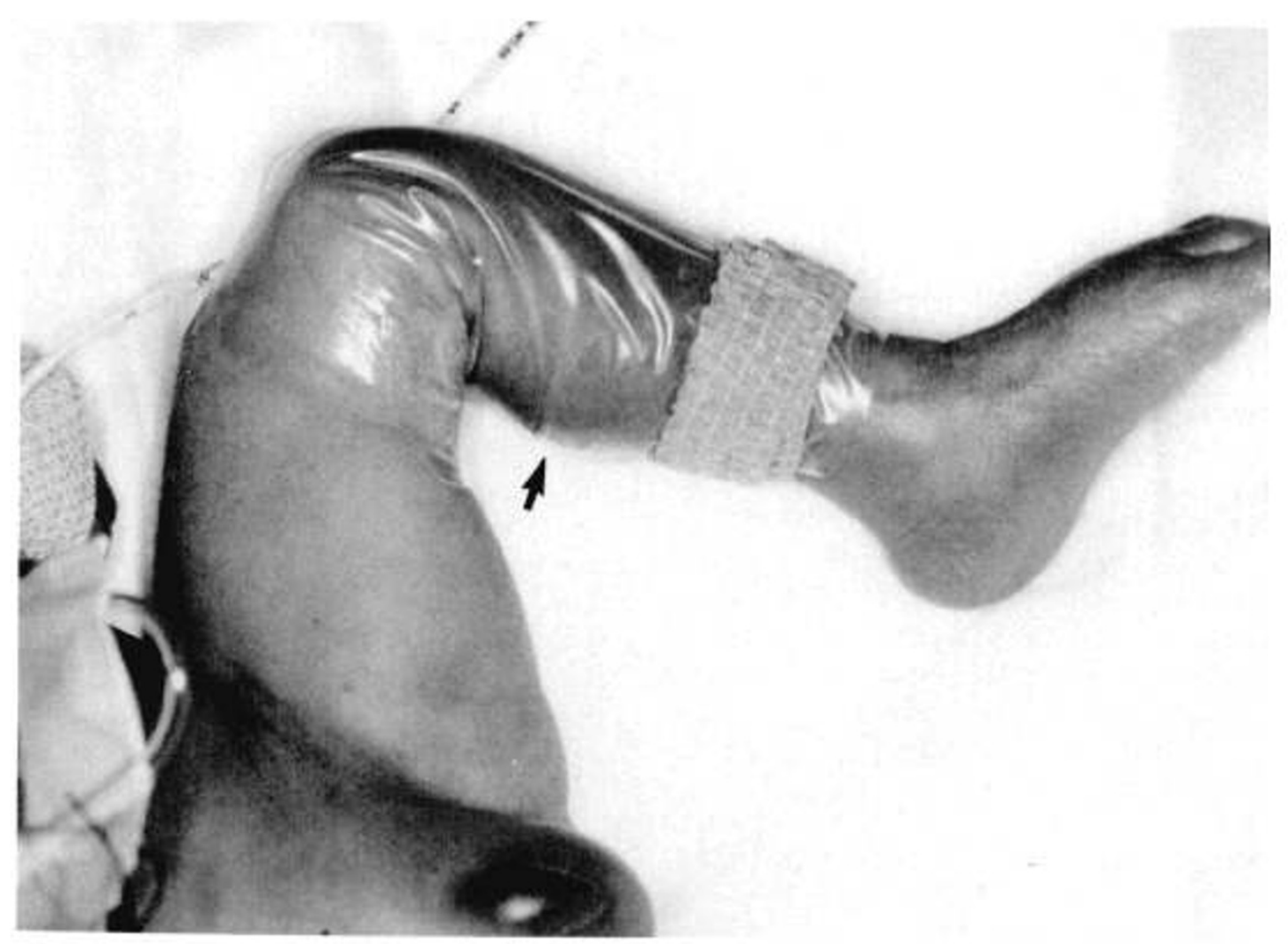

Figure 1. Treated leg of study infant, wrapped with SPD. Area used to measure TEWL is denoted by arrow. 
$500 \mathrm{mg} / \mathrm{L}$ streptomycin. The tissue was stored in this medium for less than $3 \mathrm{~h}$ before transplantation into nude mice. The HFS grafts ranged in diameter from 5 to 15 $\mathrm{mm}$.

Male nude mice (nu/nu, BALB/c) were housed in a laminar flow facility. Before each procedure, the mice were anesthetized with an intraperitoneal injection of $0.07 \mathrm{mg} / \mathrm{g}$ sterile Nembutal sodium solution (Abbott Laboratories, Chicago, IL).

Sixteen grafts from four human fetuses were transplanted subcutaneously to nude mice using the method described by Lane et al. (11). The gestational ages of the HFS grafts ranged from $18-22 \mathrm{wk}$ at the time of transplant to 23-26 wk (the "corrected EGA") at the time of exposure. Corrected EGA was calculated as the initial EGA plus the time, in weeks, lapsed between transplant and graft exposure. Each mouse received two grafts, which were placed on the superior aspect of the dorsolateral back on either side of the midline. Grafts remained buried for a minimum of $2 \mathrm{wk}$ and a maximum of $6 \mathrm{wk}$ before exposure.

The buried grafts received a second procedure $2 \mathrm{wk}$ or more after transplantation. An incision was made through the mouse skin and panniculus carnosus in the area overlying the HFS graft, and the graft was exposed to the surface. Both grafts were exposed by this procedure on the same day. Figure 2 shows a study mouse after graft exposure. One graft was treated with an SPD, the other remained uncovered. On this day (d 0$)$ and $d 2$ and 4 , measurements were made of TEWL and skin blood flow (Laser Blood Flow Monitor, Moor Instruments, Millwey, Axminster Devon, UK) on both grafts. TEWL was measured both through the dressing and $10 \mathrm{~min}$ after its temporary removal on the treated side, similar to the way it was measured in the infant trial. After all measurements were completed on d 4, biopsies of the HFS grafts were obtained for routine histology, electron microscopy, and an epidermal keratinocyte proliferation assay. The animals were then killed by cervical dislocation.

Dressings. The same nonadhesive, gas-sterilized polyurethane dressing as used in the clinical trial was used on the HFS grafts. This dressing was attached to the center of a regular piece of adhesive Bioclusive that had the center removed to accommodate the nonadhesive SPD. The dressings were gas sterilized before use. The nonadhesive portion was positioned over the HFS graft to be treated, with the remainder of the adhesive dressing affixed to the skin of the nude mouse. The other graft remained uncovered. The dressing was maintained in place by a piece of Coban self-adherent wrap that had windows created to accommodate the HFS grafts. This was secured on the ventral abdomen with two stainless steel surgical clips.

Cell proliferation assay. On d 4, biopsies were obtained of treated and control HFS grafts. Two h before biopsy, the mouse was given a $0.3-\mathrm{mL}$ intraperitoneal injection of a $4 \times 10^{3}-\mathrm{mg} / \mathrm{L}$ solution of BrdU in sterile water (Amersham International, Amersham, UK). These samples were preserved, sectioned, fixed, denatured, and incubated with a primary antibody, murine anti-BrdU IgG2a, and then a secondary antibody, peroxidase-linked IgG anti-mouse IgG2a. They were then stained. A "labeling index" was calculated for each biopsy by dividing the number of darkly staining basal keratinocytes by the total number of basal keratinocytes counted. Specimens were evaluated by an examiner who was blinded to treatment group, and at least 500 basal keratinocytes were counted per biopsy specimen. Positively staining cells in the pilosebaceous unit were not included in this evaluation.

Electron microscopy. For electron microscopy, tissues were fixed in Karnofsky's solution for $24 \mathrm{~h}$, stored in sodium cacodylate buffer in the cold, and then postfixed in either $1 \% \mathrm{OsO}_{4}$ containing $1.5 \%$ potassium ferrocyanate buffered with $0.1 \mathrm{M}$ sodium cacodylate or in $0.2 \%$ $\mathrm{RuO}_{4}$ in $0.1 \mathrm{M}$ buffer. Tissues were then processed for electron microscopy as previously described (15). $\mathrm{OsO}_{4^{-}}$ fixed tissues were additionally stained en bloc with $2.5 \%$ uranyl acetate before dehydration in acetone. Ultrathin sections were stained with lead citrate before examination in a Zeiss EM10C transmission electron microscope.

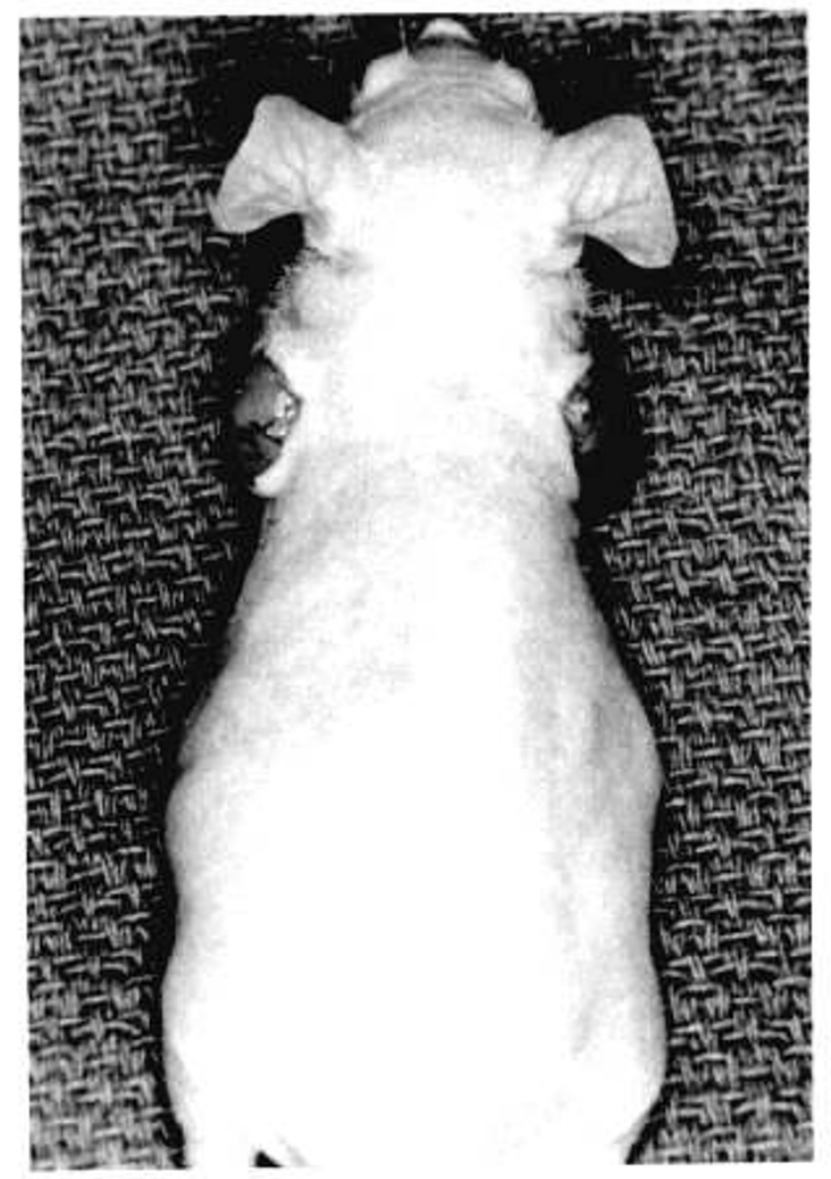

Figure 2. Nude mice had HFS grafts transplanted s.c. 2 to 6 wk before exposure. This photograph shows the dorsal appearance of a representative study animal immediately after incision of the overlying mouse skin. thereby exposing the grafts to the air. The treated side has not yet been covered with the SPD. 
The electron microscopist was blinded to the origin of the samples.

Volume densities of membrane-coating granules in $\mathrm{OsO}_{4}$-fixed tissues were determined by the pointcounting method as described by Weibel (16) from five to seven micrographs from each sample at a total magnification of $60000 \times$. At this magnification, it is possible to identify clearly and distinguish granule types. Point counts were converted into volume densities related to a reference volume of $1 \mathrm{~cm}^{3}$ of tissue.

Eight to 10 representative photographs of $\mathrm{RuO}_{4}$-fixed sections from treated and untreated sites were examined in a blinded fashion for possible differences in the morphology of the intercellular spaces of the stratum corneum.

Data analysis. Data were analyzed using paired twotailed $t$ tests.

\section{RESULTS}

Infant Study

TEWL. TEWL measurements were taken from both legs before application of the dressing to the leg to be treated. These readings were consistently similar between the two sides (within $3 \mathrm{~g} / \mathrm{m}^{2} \cdot \mathrm{h}^{-1}$ ), with mean TEWL of $24.27 \pm 16.17 \mathrm{~g} / \mathrm{m}^{2} \cdot \mathrm{h}^{-1}$ and $24.40 \pm 14.86$ $\mathrm{g} / \mathrm{m}^{2} \cdot \mathrm{h}^{-1}$, respectively, on control and treated sides. The TEWL measurements on $\mathrm{d} 0$ were identical at control and treated sites in five of the 15 study infants.

On 16 different occasions in the first four patients, TEWL was measured at the following time points after dressing removal: $2,5,10,15,20$, and $30 \mathrm{~min}$. This information was plotted with elapsed time after dressing removal on the $x$ axis and TEWL on the $y$ axis. The slope of this line was compared statistically with a slope of zero, which represented no change in measured TEWL values with respect to time. The slopes ranged from -0.0112 to +0.0938 and showed no statistically significant difference from zero when analyzed individually $(0.949>p>0.107)$ or as the average of all $16(p=0.101)$. Because these data revealed no relationship of TEWL to elapsed time after dressing removal when measured between 2 and $30 \mathrm{~min}, 10 \mathrm{~min}$ was selected as the elapsed time at which to obtain all other measurements.

To evaluate the efficacy of the SPD in decreasing water loss, TEWL of the control (untreated) leg was compared with that of the treated leg with the dressing in place. The values of TEWL ranged from 10 to $67 \mathrm{~g} / \mathrm{m}^{2} \cdot \mathrm{h}^{-1}$ before placement of the dressing to 4 to $30 \mathrm{~g} / \mathrm{m}^{2} \cdot \mathrm{h}^{-1}$ after placement of the SPD. A 57\% decrease in TEWL was found immediately after placement of the dressing on $\mathrm{d} 0(p<$ $0.001)$. The comparisons of the treated and control legs at the other time points in the study, shown in Table 1, revealed continued statistical significance daily, with less TEWL at the treated site.

To assess the effect of the SPD on epidermal barrier function, we evaluated TEWL at the treated site $10 \mathrm{~min}$ after temporary removal of the dressing on each of the
Table 1. Mean TEWL $\left(\mathrm{g} / \mathrm{m}^{2} \cdot \mathrm{h}^{-1}\right)$ of control sites $(C)$, treated sites through dressing $(R x)$, and treated sites after dressing removal $(R x W / O)$ in the infant study*

\begin{tabular}{cccc}
\hline Day & TEWL C & TEWL Rx $\dagger$ & $\begin{array}{c}\text { TEWL } \\
\text { RxW/O } \ddagger\end{array}$ \\
\hline 0 & $24.27 \pm 16.17$ & $10.60 \pm 6.99$ & $24.40 \pm 14.86$ \\
& & $(<0.001)$ & $(0.8093)$ \\
1 & $24.20 \pm 13.93$ & $9.00 \pm 5.83$ & $20.80 \pm 15.08$ \\
& $(<0.001)$ & $(<0.001)$ \\
2 & $21.40 \pm 13.73$ & $8.33 \pm 5.53$ & $18.00 \pm 14.53$ \\
& & $(<0.001)$ & $(<0.001)$ \\
4 & $17.00 \pm 8.67$ & $6.00 \pm 3.09$ & $11.87 \pm 5.79$ \\
& & $(<0.001)$ & $(<0.001)$ \\
7 & $12.40 \pm 5.96$ & $4.20 \pm 2.81$ & $8.00 \pm 4.78$ \\
& & $(<0.001)$ & $(<0.001)$ \\
\hline
\end{tabular}

* Numbers represent mean TEWL \pm SD. Numbers in parentheses represent $p$ value vs control.

$\dagger$ Measurement taken through the dressing on the treated side.

$\ddagger$ Measurement taken, on treated side, 10 min after dressing had been temporarily removed.

same days as all other measurements. As shown in Table 1 , a statistically significant decrease in TEWL $(p<0.001)$ was seen at the treated site in comparison with the control on every study day except $\mathrm{d} 0$.

To evaluate the cumulative effect of treatment on TEWL over the entire treatment period, these same data were analyzed by statistically comparing AUC, which was calculated from the graphic representation of the data as shown in Figure 3. There was a statistically significant difference between the control and treated (with the dressing in place) sites $(p<0.001)$. When control sites were compared with measurements obtained from treated skin after temporary dressing removal, AUC were again significantly lower at treated sites than control sites $(p<0.001)$.

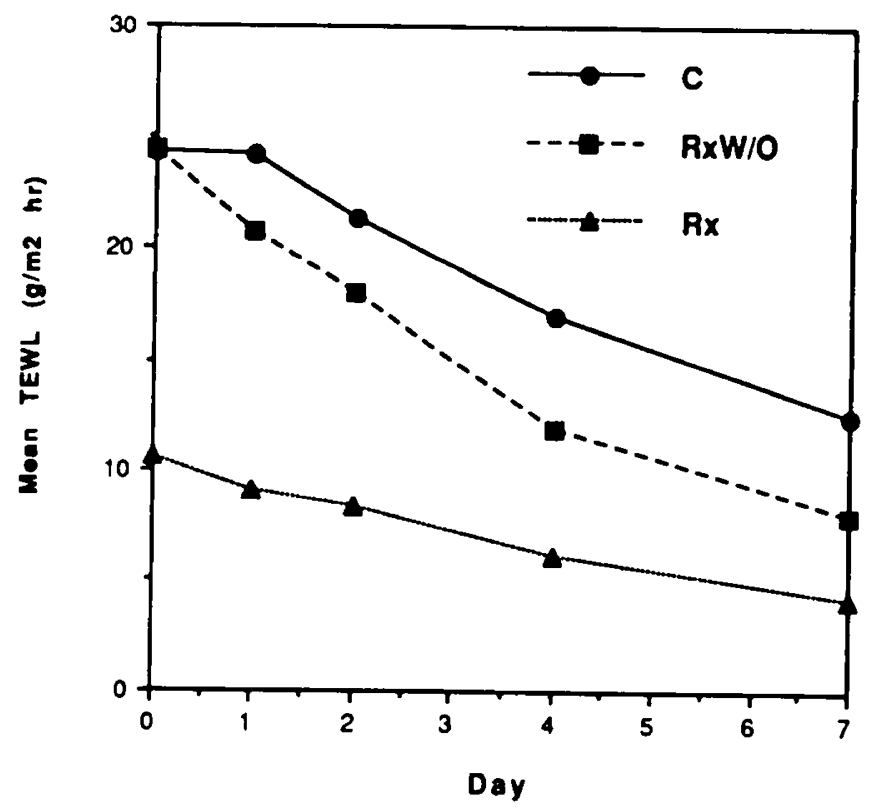

Figure 3. TEWL $\left(\mathrm{g} / \mathrm{m}^{2} \cdot \mathrm{h}^{-1}\right)$ vs day for control sites $(C)$, treated sites 10 min after dressing removal $(R \times W / O)$, and treated sites through dressing $(R x)$ in the infant study. 
Microbiology. The trend in surface density of bacteria on the skin was an increase with time at both treated and control sites. Staphylococcus spp, coagulase-negative, was by far the most common isolate in the study. As shown in Table 2 , the mean $\log _{10}$ colony-forming units per $\mathrm{cm}^{2}$ of coagulase-negative staphylococci was consistently less at the treated versus control sites, with a statistically significant decrease on $\mathrm{d} 4(p=0.022)$. To statistically compare bacterial numbers at control and treated sites over time, AUC were again compared. The average AUC at treated sites was significantly lower than the average AUC at control sites $(6.46+7.22$ versus $11.44+6.82)(p<0.001)$. There was no $M$. furfur or Candida spp isolated in any of these patients. Two patients had coagulase-negative staphylococci isolated from the blood, one on d 14 of life and the other on $\mathrm{d} 7$. In both cases, the positive cultures were reported on the last day of treatment with the SPD (one patient was treated with the dressing for $14 \mathrm{~d}$, the other for $7 \mathrm{~d}$ ). Both patients were treated with a second course of antibiotics for these positive cultures, and both did well clinically. One patient had coagulase-negative staphylococci cultured from an i.v. catheter tip but not the blood. This patient was also treated with a second course of antibiotics and also did well.

\section{Animal Study}

TEWL. TEWL measurements were taken from both grafts before application of the dressing to the side to be treated. These readings were consistently similar between the two sides (within $4 \mathrm{~g} / \mathrm{m}^{2} \cdot \mathrm{h}^{-1}$ ), with mean $\mathrm{d} 0$ TEWL of $36.87 \pm 14.45 \mathrm{~g} / \mathrm{m}^{2} \cdot \mathrm{h}^{-1}$ and $37.00 \pm 15.19$ $\mathrm{g} / \mathrm{m}^{2} \cdot \mathrm{h}^{-1}$ on control and treated sides, respectively.

To evaluate the efficacy of the SPD in decreasing water loss, TEWL of the control (untreated) graft was compared with that of the treated graft with the dressing in place. The values of TEWL ranged from $12-56 \mathrm{~g} / \mathrm{m}^{2} \cdot \mathrm{h}^{-1}$ before placement of the dressing to $3-15 \mathrm{~g} / \mathrm{m}^{2} \cdot \mathrm{h}^{-1}$ after placement of the dressing. There was a $73 \%$ mean decrease in TEWL immediately after placement of the dressing on $\mathrm{d} 0(p<0.001)$. On $\mathrm{d} 2$ and 4 , there was a continued statistically significant decrease in TEWL at the treated site (Table 3 ).

To assess the effect of the SPD on epidermal barrier function, we evaluated the TEWL at the treated site 10 min after temporary removal of the dressing on $\mathrm{d} 2$ and 4.

Table 2. Mean $\log _{10}$ colony-forming units $\pm S D$ of coagulase-negative staphylococci at control $(C)$ and treated $(R x)$ sites in the infant study*

\begin{tabular}{crccc}
\hline Day & $n$ & $\mathrm{C}$ & $\mathrm{Rx}$ & $p$ value \\
\hline 0 & 15 & $0.48 \pm 0.63$ & $0.33 \pm 0.59$ & 0.429 \\
1 & 9 & $1.25 \pm 1.69$ & $0.32 \pm 0.48$ & 0.163 \\
2 & 9 & $1.25 \pm 1.27$ & $0.51 \pm 0.82$ & 0.144 \\
4 & 15 & $1.70 \pm 1.54$ & $0.98 \pm 1.60$ & 0.022 \\
7 & 15 & $2.64 \pm 1.48$ & $1.74 \pm 1.95$ & 0.069
\end{tabular}

${ }^{*} n=$ Number of patients represented; in the last six patients, frequency of cultures was decreased to $d 0,4$ and 7 only.
Table 3. Mean TEWL $\left(\mathrm{g} / \mathrm{m}^{2} \cdot \mathrm{h}^{-l}\right)$ of control (C) HFS grafts, HFS grafts treated through dressing $(R x)$, and HFS grafts treated after dressing removal $(\mathrm{RxW} / \mathrm{O})^{*}$

\begin{tabular}{cccc}
\hline Day & TEWL C & TEWL Rx $\dagger$ & $\begin{array}{c}\text { TEWL } \\
\text { RxW/O } \ddagger\end{array}$ \\
\hline 0 & $36.87 \pm 14.45$ & $10.00 \pm 4.34$ & $37.00 \pm 15.19$ \\
& & $(<0.001)$ & $(0.875)$ \\
2 & $21.50 \pm 6.41$ & $5.75 \pm 2.87$ & $18.13 \pm 8.44$ \\
& & $(<0.001)$ & $(0.233)$ \\
4 & $19.71 \pm 5.59$ & $2.43 \pm 0.98$ & $10.71 \pm 5.96$ \\
& & $(<0.001)$ & $(0.005)$ \\
\hline
\end{tabular}

* Numbers represent mean TEWL \pm SD. Numbers in parentheses represent $p$ value $i s$ control.

$\dagger$ Measurement taken through the dressing on the treated graft.

$\ddagger$ Measurement taken, on treated graft, $10 \mathrm{~min}$ after dressing had been temporarily removed.

As shown in Table 3, there was a consistently lower TEWL at the treated site on both days, with a statistically significant decrease $(p=0.005)$ on $\mathrm{d} 4$.

To evaluate the cumulative effect of treatment on TEWL over the 4-d treatment period, the data were analyzed by statistically comparing AUC as performed in the infant study. This analysis, which included only seven of the animals secondary to one death on study d 2, revealed a lower AUC at both the treated site with the dressing in place and the treated site after temporary dressing removal compared with the control site. This decrease was statistically significant on the treated site with the dressing in place $(p<0.001)$ and approached statistical significance on the treated site after temporary dressing removal ( $p=0.063$ ).

Skin blood flow. Skin blood flow analysis via laser Doppler was performed on each study day at both treated and control sites. No statistically significant differences were seen on $\mathrm{d} 0$ and 2 , but there was significantly lower skin blood flow in the control grafts on $\mathrm{d} 4(p=0.01)$ (Table 4).

Keratinocyte proliferation. Basal keratinocyte proliferation, as analyzed with a BrdU assay on study d 4, revealed a nearly 2 -fold increase in labeling index in the treated skin compared with control skin $(1.99 \pm 0.66 \%$ versus $1.09 \pm 0.19 \%)(p=0.01)$.

Histology/electron microscopy. Histologic analysis by a blinded examiner revealed no consistent differences between treated and control grafts with respect to the stratum corneum or stratum malpighii. Electron microscopic analysis of membrane-coating granules in four paired samples (four control and four treated) showed volume densities of $8.4+3.6 \mathrm{~mm}^{3} / \mathrm{cm}^{3}$ and $9.0+3.4$ $\mathrm{mm}^{3} / \mathrm{cm}^{3}$ in the control and treated samples, respec-

Table 4. Mean laser Doppler blood flow (flux) of control (C) and treated $(R x)$ HFS grafts*

\begin{tabular}{cccc}
\hline Day & Flux C & Flux Rx & $p$ value \\
\hline 0 & $210.29 \pm 50.12$ & $204.86 \pm 32.18$ & 0.766 \\
2 & $164.14 \pm 10.46$ & $183.00 \pm 39.37$ & 0.200 \\
4 & $166.50 \pm 39.28$ & $200.29 \pm 29.46$ & 0.01 \\
\hline
\end{tabular}

* Numbers represent mean flux $\pm \mathrm{SD}$. 
tively. The two-tailed $p$ value was 0.8647 ; therefore, the numbers were not significantly different. The morphology of the membrane coating granules in control and treated sites was the same. Examination of the $\mathrm{RuO}_{4}$ fixed stratum corneum from control and treated sites did not show any obvious morphologic differences. The intercellular spaces of all samples demonstrated patterned lipid lamellae and morphologic features identical with those previously described in mammalian stratum corneum $(15,17)$.

\section{DISCUSSION}

Premature infants experience excessive fluid losses through the skin as a result of immaturity of the epidermal permeability barrier (4). These fluid losses can contribute to a hyperosmolar hypernatremic state (7), which may be related to the increased risk of intracranial hemorrhage in this patient population (18). Replacement of fluid losses results in rapid shifts in intravascular volume, exacerbating such conditions as patent ductus arteriosus, congestive heart failure, and pulmonary edema (7). These infants are at increased risk for hypothermia (7), and skin evaporative heat loss may account for up to $20 \%$ of the energy expenditure of infants under $30 \mathrm{wk}$ gestation (19).

TEWL is a measure of the effectiveness of the epidermal barrier and can be used to quantitate epidermal maturity (4). TEWL decreases as a premature infant ages; by 2 wk postnatal age, the barrier function is similar to that of a term infant (3). It is within these first $2 \mathrm{wk}$ of life that large shifts in intravascular volume may take place.

The accelerated rates of healing in dressing-treated wounds reported by Winter in 1962 (5) were replicated in human subjects 1 y later by Hinman et al. (20). Eaglstein (21) has suggested five possible mechanisms of these effects: 1) easier migration of epithelial cells, 2) increased partial pressure of oxygen, 3) increased local concentration of growth factors, 4) favorable effects of increased microbial flora, and 5) maintenance of an electrical potential between the wounded skin and the surrounding normal skin. The effect that occlusion has on wounds appears not to be limited to the epidermis (22), with variable observations having been reported in the dermis, from a diminution of neutrophils and fibroblasts (23) to an earlier appearance of dermal mononuclear cells and more numerous fibroblasts and collagen (24).

Although the skin compromise seen in premature infants is primarily limited to epidermis and stratum corneum, it has been hypothesized that these patients might similarly benefit from SPD, and several investigators have studied their use in these patients. Knauth et al. (7) found an acute decrease in TEWL with the dressing in place, but no difference between untreated skin and treated skin with the dressing removed after $5 \mathrm{~d}$ of treatment. Bustamante and Steslow (6) treated $40-60 \%$ of the body surface of six premature infants with dressings and again found a decreased TEWL with the dressing in place, as well as a decrease in consumed electric current by the radiant warmer in the treatment group compared with untreated controls. Vernon et al. (8) found a decrease in TEWL on treated sites with the dressing in place, an effect that decreased in magnitude with time. Similar results were reported by Stuart et al. (9).

A concern in treating patients with these dressings has been the possibility of an increase in bacteria or fungi beneath the dressing, as reported in 1984 by Mertz and Eaglstein (25), who found an increased number of organisms and a shift toward Gram-negative predominance in superficial wounds in a Yorkshire pig treated with a wound dressing. Because premature infants are predisposed to systemic infection with skin-associated organisms such as coagulase-negative staphylococci (26) and M. furfur (27), recent investigators have addressed this important issue by evaluating changes in microflora in premature infants treated with wound dressings. Vernon et al. (8) treated the anterior thorax or thigh of 10 patients and found an increase in bacterial number with time, with coagulase-negative staphylococci predominating, at both treated and untreated sites, but no statistically significant differences in bacterial density between the two sites. Elaborating on the microbiologic sequelae of treatment with wound dressings, Stuart et al. (9) evaluated not only bacterial number but species, resistance patterns, slime production, and plasmid analysis in 13 treated premature infants. They again found no significant difference in bacterial number between treated and untreated sites. In addition, they found an increase in antibiotic resistance with time at both sites and no difference in slime production or plasmid analysis. There was no significant difference in $M$. furfur or Candida spp recovery between the two sites.

The semipermeable nature of the membrane is of paramount importance in the treatment of these patients. Studies using vapor impermeable dressings such as vinylidene polymer plastic film (Saran Wrap) have demonstrated several-thousand-fold increases in microbial flora at the skin sites treated with the film (28). Also, Proksch et al. (29) have shown that impermeable dressings, by providing an artificial barrier, suppressed the burst in DNA synthesis normally seen in hairless mouse skin that has been treated with acetone to disrupt the permeability barrier. Rovee et al. (30) studied the effects of occlusion with an impermeable dressing on mitotic response of tape-stripped wounds and found a decreased number of mitoses at occluded sites. Proksch et al. (31) also showed that impermeable dressings applied after acute acetoneinduced barrier disruption suppress the normal increase in lipid synthesis, another factor that may affect restoration of barrier function. When SPD were applied, there was partial suppression of the burst in DNA synthesis (29) and slight to no inhibition of the TEWL recovery (32) in comparison with that seen in uncovered skin. These studies would suggest that the use of artificial dressings on barrier-disrupted skin might delay barrier restoration, 
an effect whose magnitude is related to the impermeability of the dressing.

Our results from both the infant and animal trials again support the marked reduction in TEWL seen with an SPD in place. Furthermore, we have shown that there is an improvement in the barrier function of premature infant skin when the skin is treated with the dressings. This conclusion is supported by results from both our human and animal trials. The biologic basis for the improvement in barrier function is unclear but perhaps represents a response unique to premature infant skin. Electron microscopy did not reveal any differences in membrane-coating granules or the stratum corneum intercellular spaces to account for the improved TEWL, although subtle changes in morphology or changes in the lipid composition of the lamellae would not be detected. Our microbiologic findings support the notion that there is no increased colonization with bacteria or fungi underneath the dressings, but the decreased bacterial counts seen at treated sites might be related to decreased handling of the SPD-treated limb by physician and nursing staff. Hence, although our data do not demonstrate increased propensity of bacterial or fungal proliferation under occlusion, the decreased numbers demonstrated at these sites must be interpreted with caution.

The finding of increased cellular proliferation in treated skin is interesting. As mentioned above, Proksch $e t$ al. (29) showed that barrier abrogation results in a burst in epidermal DNA synthesis and that restoration of barrier function with dressings suppresses this expected burst. The amount of suppression of DNA synthesis was directly correlated with the vapor impermeability of the dressing. Therefore, we might have expected to see decreased DNA synthesis in the treated fetal skin graft compared with the untreated graft. This disparity in results may reflect differences in the models of barrier disruption used in the studies, i.e. a difference in the response of fetal skin to barrier disruption. Perhaps the TEWL in premature infants is so high that it suppresses DNA proliferation and barrier recovery rather than serving as a stimulus for this response.

Skin blood flow analysis in our study revealed decreased blood flow at control sites with relatively stable blood flow at treated sites over the 4-d study period. Wu et al. (33) demonstrated that premature infants have lower peripheral vascular resistance and increased cutaneous blood flow than term infants at birth and found an incremental increase in resistance and decrease in skin blood flow with advancing postnatal age, regardless of birth weight or gestational age. This "expected" decrease in blood flow was seen in our control grafts. Our expectation of a greater decrease in cutaneous blood flow at treated sites, based on the hypothesis that with accelerated maturation these grafts would require less blood supply, was not substantiated. It has recently been demonstrated that with local warming of the skin of both term and premature neonates there is a concomitant increase in cutaneous blood flow (with a decrease in flow seen with local cooling) (34), presumably related to local myogenic responses. Perhaps in our study the normal decrease in cutaneous blood flow was offset by a local increase in temperature under occluded skin sites, resulting in this myogenically mediated reactive hyperemia.

One finding noted in several infants was drying and fissuring of uncovered skin. Treated skin appeared well hydrated throughout the study period and for the first 1 to $2 \mathrm{~d}$ after dressing removal, but after this time began to show some signs of dryness as well. These skin changes can themselves result in increased water loss, and the hydration of microscopic cracks and fissures might partially explain the marked effect seen with wound dressing treatment. A recent study that looked at repeated application of emollient cream to premature infants' skin demonstrated statistically less dermatitis of the treated skin (35). Perhaps there exists an optimal combination treatment strategy of early treatment with SPD followed by immediate and meticulous application of emollients to further maintain barrier function.

We conclude that treatment with SPD not only greatly decreases TEWL while in place but also improves the barrier function of treated skin compared with control (untreated) skin. This study supports further trials of SPD in treating larger surface areas of these infants, in which case other parameters such as weight loss or gain, fluid requirements, electrolyte balance, metabolic rate, thermoregulation, incidence of sepsis or other complications, and overall well being can be evaluated. We are studying further the extent to which proliferation, differentiation, skin blood flow, and changes in the epidermal lipid profile are involved in the improved barrier function seen in premature infant skin treated with an SPD.

Acknowledgments. The authors thank Byron W. Brown, Jr. for statistical assistance, Donald C. Swartzendruber for performing the electron microscopy, and Philip W. Wertz for the statistical analysis of the membranecoating granule counts.

\section{REFERENCES}

1. Rutter N 1988 The immature skin. Br Med Bull 44:957-970)

2. West DP, Worobec S, Solomon LM 1981 Pharmacology and toxicology of infant skin. J Invest Dermatol 76:147-150

3. Harpin VA, Rutter N 1983 Barrier properties of the newborn infant's skin. J Pediatr 102:419-425

4. Hammarlund K, Sedin G 1979 Transepidermal water loss in newborn infants. III. Relation to gestational age. Acta Paediatr Scand 68:795-801

5. Winter GD 1962 Formation of the scab and the rate of epithelialization of superficial wounds in the skin of the young domestic pig. Nature 193:293-294

6. Bustamante SA. Steslow J 1989 Use of a transparent adhesive dressing in very low birthweight infants. J Perinatol 9:165-169

7. Knauth AK. Gordin M, McNelis W, Baumgart S 1989 Semipermeable polyurethane membrane as an artificial skin for the premature neonate. Pediatrics 83:945-950

8. Vernon HJ, Lane AT, Wischerath LJ, Davis JM. Menegus MA 1990 Semipermeable dressing and transepidermal water loss in premature infants. Pediatrics $86: 835-847$

9. Stuart SM, Lane AT, Mickelson PA 1991 Semipermeable dressings on premature infant's skin reduce bacterial colonies and do not alter bacterial type. J Invest Dermatol 96:573(abstr)

10. Silverman RA, Lender J, Elmets CA 1989 Effects of occlusive and semiocclusive dressings on the return of barrier function to transepidermal water loss in standardized human wounds. J Am Acad Dermatol 20:755-760 
11. Lane AT, Scott GA, Day KH 1989 Development of human fetal skin transplanted to the nude mouse. J Invest Dermatol 93:787-791

12. Evans NJ, Rutter N 1986 Development of the epidermis in the newborn. Biol Neonate 49:74-80

13. Dubowitz LMS, Dubowitz V, Goldberg C 1970 Clinical assessment of gestational age in the newborn infant. J Pediatr 77:1-10

14. Mercer BM, Sklar S, Shariatmadar A, Gillieson MS, D'Alton ME 1987 Fetal foot length as a predictor of gestational age. Am J Obstet Gynecol 156:350 355

15. Madison KC, Swartzendruber DC, Wertz PW, Downing DT 1987 Presence of intact intercellular lamellae in the upper layers of the stratum corneum. J Invest Dermatol 88:714-718

16. Weibel ER 1979 Stereological Methods. Academic Press, London, pp 101-161

17. Swartzendruber DC, Wertz PW, Kitko DJ, Madison KC. Downing DT 1989 Molecular models of the intercellular lipid lamellae in mammalian stratum corneum. J Invest Dermatol 92:251-257

18. Thomas DB 1976 Hyperosmolality and intraventricular hemorrhage in premature babies. Acta Paediatr Scand 65:429_432

19. Maurer A, Micheli JL, Schuetz Y, Freymond D, Jequier E 1984 Transepidermal water loss and resting expenditure in preterm infants. Helv Paediatr Acta 39:405-418

20. Hinman CC, Maibach H, Winter GD 1963 Effect of air exposure and occlusion on experimental human skin wounds. Nature $2(0): 377-378$

21. Eaglstein WH 1984 Effects of occlusive dressings on wound healing. Clin Dermatol 2:107-111

22. Falanga V 1988 Occlusive wound dressings: why, when, which? Arch Dermatol 124:872-877

23. Linsky CB, Rovee DT, Dow T 1981 Effect of dressings on wound inflammation and scar tissue. In: Dineen P, Hildick-Smith G (eds) The Surgical Wound. Lea \& Febiger, Philadelphia, pp 191-206
24. Winter GD 1972 Epidermal regeneration studied in the domestic pig. In: Maibach HL, Rovee DT (eds) Epidermal Wound Healing. Year Book Medical Publishers, Chicago, pp $71-112$

25. Mertz PM, Eaglstein WH 1984 The effect of a semiocclusive dressing on the microbial population in superficial wounds. Arch Surg 119:287-289

26. Baumgart S, Hall SE, Campos JM. Polin RA 1983 Sepsis with coagulasenegative staphylococci in critically ill newborns. Am J Dis Child 137:461-46.

27. Aschner JL, Punsalang A, Maniscalco WM, Menegus MA 1978 Percutaneous central venous catheter colonization with Malassezia furfur: incidence and clinical significance. Pediatrics 80:535-539

28. Aly R, Shirley C. Cunico B, Maibach HI 1978 Effect of prolonged occlusion on the microbial flora, $\mathrm{pH}$, carbon dioxide and transepidermal water loss on human skin. J Invest Dermatol 71:378-381

29. Proksch E, Feingold KR, Mao-Qiang M, Elias PM 1991 Barrier function regulates epidermal DNA synthesis. J Clin Invest 87:1668-1673

30. Rovee DT, Kurowsky CA, Labun J 1972 Local wound environment and epidermal healing: mitotic response. Arch Dermatol 106:330-334

31. Proksch E, Holleran WM, Menon GK, Elias PM, Feingold KR 1993 Barrier function regulates epidermal lipid and DNA synthesis. Br J Dermatol 128:473-482

32. Grubauer G, Elias PM, Feingold KR 1989 Transepidermal water loss: the signal for recovery of barrier structure and function. J Lipid Res 30:323-333

33. Wu PY, Wong WH, Guerra G, Miranda R, Godoy RR, Preston B, Schoentgen $S$, Levan NE 1980 Peripheral blood flow in the neonate. I. Changes in total, skin, and muscle blood flow with gestational and postnatal age. Pediatr Res 14:1374-1378

34. Beinder E, Trojan A, Bucher HU. Huch A, Huch R 1994 Control of skin blood flow in pre- and full-term infants. Biol Neonate $65: 7-15$

35. Lane AT, Drost SS 1993 Effects of repeated application of emollient cream to premature infants' skin. Pediatrics 92:415-419 